\title{
Study of photon-tagged jet events in high-energy heavy ion collisions with CMS
}

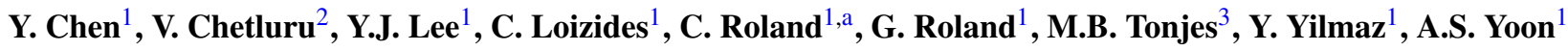 \\ ${ }^{1}$ MIT, Cambridge, MA, USA \\ ${ }^{2}$ University of Illinois, Chicago, IL, USA \\ ${ }^{3}$ University of Maryland, College Park, MD, USA
}

Received: 29 August 2008 / Revised: 2 February 2009 / Published online: 27 March 2009

(C) Springer-Verlag / Società Italiana di Fisica 2009

\begin{abstract}
The energy loss of fast partons traversing the strongly interacting matter produced in high energy nuclear collisions is one of the most interesting observables to probe the nature of the produced medium. The collisional and radiative energy loss of the partons will modify the fragmentation functions depending on the path length in the medium. $\mathrm{Pb}+\mathrm{Pb}$ collisions at $\sqrt{s_{N N}}=5500 \mathrm{GeV}$ at the LHC will allow detailed measurements of the in-medium modifications of fragmentation functions of parton initiated jets, using the $\gamma$-jet channel. Since the photon does not strongly interact with the medium, the initial transverse energy of the fragmenting parton can be related to the photon transverse energy. This in turn allows for precision studies of the fragmentation function underlying these jets.
\end{abstract}

\section{Introduction}

One of the key results from the study of high energy nuclear collisions at the Relativistic Heavy-Ion Collider (RHIC) is the observation of a strong suppression of hadron yields at transverse momenta $p_{\mathrm{T}}>4 \mathrm{GeV} / c$, compared to expectations based on $p+p$ and $d+\mathrm{Au}$ collisions at the same collision energies [1-4]. For the most central $\mathrm{Au}+\mathrm{Au}$ collisions, the yield of e.g. $\pi^{0}$ 's per nucleon-nucleon collision is found to be reduced by a factor of 5 compared to the $p+p$ reference. This observation has been termed "jet-quenching" and is interpreted as a consequence of the energy loss of fast partons as they traverse the dense and hot medium produced in these collisions. The extreme magnitude of the suppression effect makes a quantitative interpretation of the result difficult, as the observed remaining yield of high- $p_{\mathrm{T}}$ hadrons is dominated by emission from the surface of the collision region and therefore carries little direct information about

a e-mail: rolandg@mit.edu the medium and the mechanisms of collisional and radiative energy loss of the partons.

$\mathrm{Pb}+\mathrm{Pb}$ collisions at $\sqrt{s_{N N}}=5500 \mathrm{GeV}$ at the LHC will allow a much more detailed exploration of the jet quenching phenomenon, due to the much larger cross-sections for hard parton-parton scattering and the access to probes like jets and isolated photons at much higher transverse momenta.

The energy loss of the partons as they traverse the medium will lead to a modification of the effective parton fragmentation functions, depending on the conditions achieved in the collision and the path length in the medium. The fragmentation functions can studied using the $\gamma$-jet channel. Since the $\gamma$ does not strongly interact with the medium, the initial transverse energy of the fragmenting parton can be determined from the $\gamma$ transverse energy [5]. Figure 1.1 shows the transverse energy correlation (top panel) and balance (bottom panel) between the isolated photon and the associated parton in $\gamma$-jet events. There is a tight correlation between the photon transverse energy and the transverse energy of the associated parton.

The top panel of Fig. 1.2 shows the jet fragmentation function on generator level constructed using the true transverse energy of the parton (open symbols) and using the associated photon $E_{\mathrm{T}}$ (closed symbols). The bottom panel of Fig. 1.2 shows the ratio of these two fragmentation functions. Using the transverse energy of the photon, the fragmentation function of the away-side parton can be measured with high precision.

The studies in this report are performed for two scenarios of the parton energy loss in $\mathrm{Pb}+\mathrm{Pb}$ collisions at the LHC: The "unquenched" scenario, using the PYTHIA event generator, does not include parton energy loss. To simulate a scenario including jet-quenching, we use the PYQUEN model [6], which has been tuned based on the results seen at RHIC. Results from a full reconstruction of the parton fragmentation functions for simulated events with both scenarios are presented and compared to the true fragmentation 

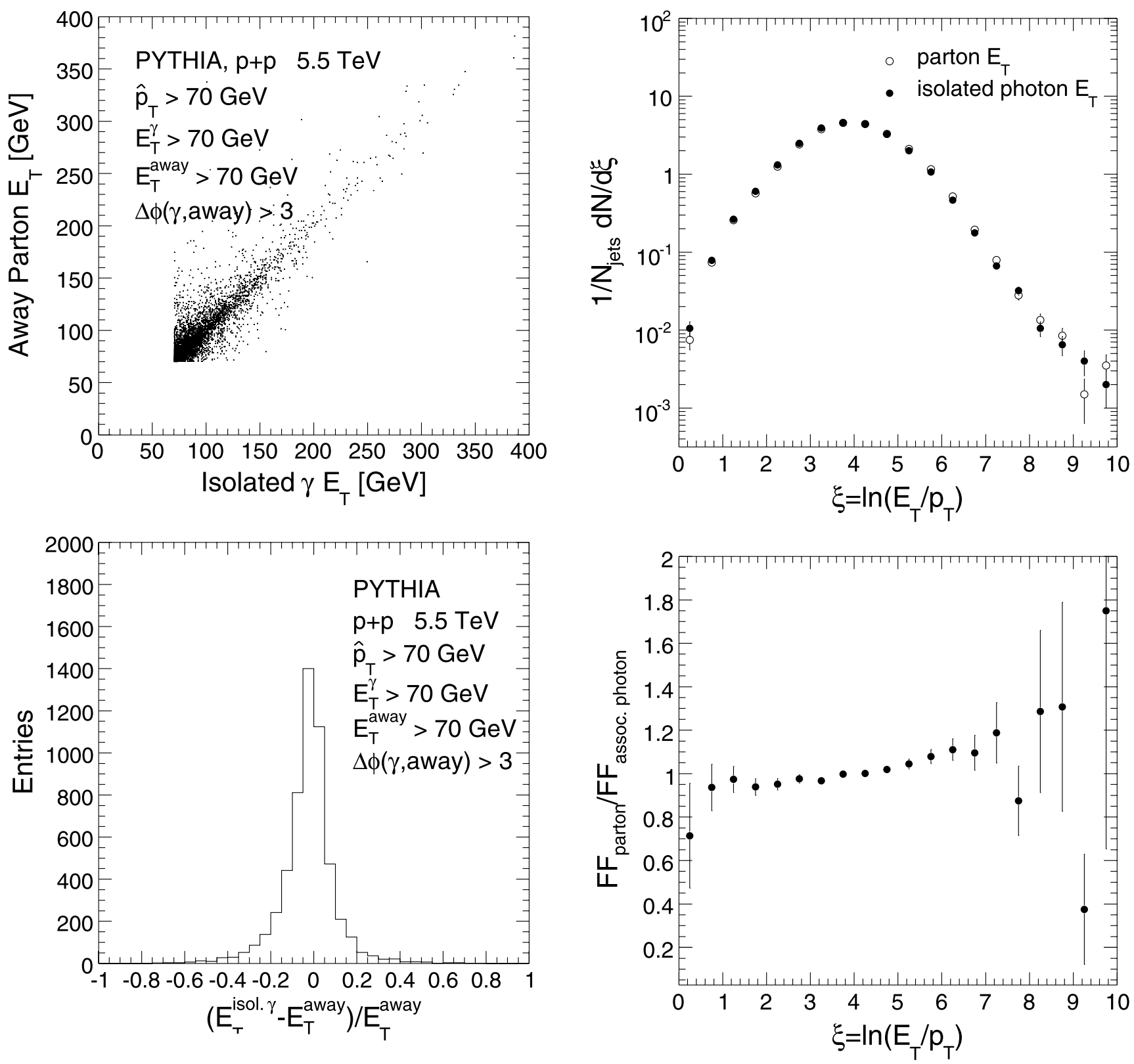

Fig. 1.1 Transverse energy correlation (top panel) and balance (bottom panel) between isolated photons and the associated away-side parton for $p+p$ events generated with the PYTHIA event generator requiring a minimum momentum transfer of $70 \mathrm{GeV}$. Partons and photons are selected to have a transverse energy above $70 \mathrm{GeV}$ and an opening angle in the transverse plane of larger than 3 radian

functions in both cases. The comparison shows that the expected statistical and systematic uncertainty of this measurement is small compared to the range of possible mediummodifications of the fragmentation functions.

\section{Simulation of the $\gamma$-jet channel in $\mathrm{Pb}+\mathrm{Pb}$ collisions}

The event simulation for this study uses the PYTHIA and PYQUEN event generators to model selected QCD (signal

Fig. 1.2 Top panel: Fragmentation function constructed using the parton energy (open symbols) and using the energy of the associated photon (filled symbols) in $\gamma$-jet events with a $\gamma$ with $E_{\mathrm{T}}>70 \mathrm{GeV}$. Bottom panel: Ratio of these two fragmentation functions

and background) channels and the HYDJET event generator to model the underlying corresponding $\mathrm{Pb}+\mathrm{Pb}$ events. For the purpose of this study we assume the standard integrated luminosity of $0.5 \mathrm{nb}^{-1}$ and a $7.8 \mathrm{~b}$ inelastic $\mathrm{Pb}+\mathrm{Pb}$ cross section, giving a total of $3.9 \times 10^{9} \mathrm{~Pb}+\mathrm{Pb}$ collisions. The study is performed for central $\mathrm{Pb}+\mathrm{Pb}$ events defined as the $10 \%$ most central collisions selected by the impact parameter of the $\mathrm{Pb}$ nuclei in the HYDJET generator. 
Table 2.1 ECAL cluster candidate rates for a nominal $\mathrm{LHC} \mathrm{Pb}+\mathrm{Pb}$ run

\begin{tabular}{lcllrrrrr}
\hline Data set & $p_{\text {T }}$ cut $[\mathrm{GeV} / c]$ & Isolated $\gamma$ & Signal $\gamma$ & $\pi^{0}$ & \multicolumn{1}{c}{$\pi_{c h}$} & \multicolumn{1}{c}{$\eta$} & $\eta^{\prime}$ \\
\hline unquenched & $>70$ & 6531 & 4288 & 23675 & 47421 & 12267 & 8194 & 30601 \\
unquenched & $>100$ & 1841 & 1216 & 4422 & 9103 & 2357 & 1567 & 5975 \\
quenched & $>70$ & 6512 & 4209 & 7569 & 14616 & 3825 & 2445 & 9235 \\
quenched & $>100$ & 1860 & 1212 & 1562 & 3000 & 829 & 515 & 2051 \\
\hline
\end{tabular}

\subsection{Isolated photon definition}

In principle, a photon that is supposed to be useful as a tag of the away-side jet energy in our analysis should be a prompt photon that is directly produced by the hard scattering of two partons, and that is not from secondary decays or the radiation product of initial or final state radiation, and ideally emerges back-to-back to the away-side parton or jet.

To preferentially select such photons, we define an isolation cone of $\Delta R<0.5$ around the photon position in pseudo-rapidity and azimuth. We then loop over the generated particles inside the cone, excluding muons and neutrinos, and calculate the total transverse momentum $\left(P_{\mathrm{T}}^{\text {tot }}\right)$ inside the cone. We check the highest transverse momentum carried by a hadron inside this cone which is denoted by $P_{\mathrm{T}}^{\max }$. An isolated photon candidate with transverse energy of $E_{\mathrm{T}}^{\gamma}$ should then satisfy the following conditions [7]

1. $P_{\mathrm{T}}^{\text {tot }}-E_{\mathrm{T}}^{\gamma}<5 \mathrm{GeV}+0.05 E_{\mathrm{T}}^{\gamma}$,

2. $P_{\mathrm{T}}^{\max }<4.5 \mathrm{GeV}+0.025 E_{\mathrm{T}}^{\gamma}$.

As mentioned above, the photons useful to tag the energy of the away-side parton are the ones emerging back-to-back. This topology is disturbed by initial and final state radiation, and so one typically applies a cut on the angle between the isolated photon and the emerging parton (or jet),

$\Delta \phi(\gamma$, jet $)>\Delta \Phi$

where we typically use $\Delta \Phi=3$ called "back-to-back criterion" in Sect. 4.

\subsection{Rate and background estimates}

To estimate the yield of $\gamma$-jet events the cross section obtained by PYTHIA is scaled by the number of binary nucleon-nucleon collisions reported by the HYDJET generator for the $10 \%$ most central $\mathrm{Pb}+\mathrm{Pb}$ collisions of $N_{\text {coll }}=$ 1578.

The dominant background to prompt photons reconstructed in the electromagnetic calorimeters are high $p_{\mathrm{T}}$ photons originating from decays of $\pi^{0}, \eta, \eta^{\prime}$ and $\omega$ mesons and photons directly originating from the fragmentation processes of high- $E_{\mathrm{T}}$ quarks and gluons.

We simulated a data set is created that corresponds to one month of $\mathrm{Pb}+\mathrm{Pb}$ data taking with an expected integrated luminosity of $0.5 \mathrm{nb}^{-1}$. The estimated rates of background events per candidate particle type are listed in Table 2.1.

This data set is then processed through the full GEANT based detector simulation and only at the level of the detector simulation is it decided whether a candidate particle actually decays into photons producing a valid signal in the calorimeters or not.

For the analysis, $\mathrm{Pb}+\mathrm{Pb}$ underlying events are simulated using the HYDJET event generator and combined with the signal events at the level of GEANT simulated hits and the combined signals are then processed by the simulated detector digitization, if in the signal event at least one ECAL cluster above $60 \mathrm{GeV}$ has been reconstructed.

\section{Event reconstruction}

\subsection{Jet reconstruction}

Jet reconstruction in heavy-ion collisions in CMS is performed with an iterative cone algorithm modified to subtract the underlying soft background on an event-by-event basis [8]. The algorithm is a variant of an iterative "noise + pedestal subtraction" technique. The performance of this algorithm in documented in [9].

\subsection{Charged particle reconstruction}

Charged particles are reconstructed using the CMS Silicon Tracking System. A detailed description of the CMS Tracking System can be found elsewhere [11]. For heavy ions, an algorithm based on seeding from the three dimensional hits of the silicon pixel detector (which has a geometrical acceptance of $80 \%$ ), an extension of the standard tracking algorithm used for $p+p$, is employed [10]. In this highmultiplicity environment, an algorithmic tracking efficiency of $\approx 70 \%$ is achieved near midrapidity with less than a few percent fake track rate for $p_{\mathrm{T}}>1 \mathrm{GeV} / c$. Tracks are reconstructed with excellent momentum resolution, $\Delta p_{\mathrm{T}} / p_{\mathrm{T}}<$ $1.5 \%$ for $p_{\mathrm{T}}<100 \mathrm{GeV} / c$. The resolution of the track impact parameter at the event vertex is less than $50 \mu \mathrm{m}$, and improves to $20 \mu \mathrm{m}$ at high $p_{\mathrm{T}}$ (above $10 \mathrm{GeV} / c$ ). 


\subsection{Photon reconstruction}

Photon reconstruction is done in three steps. First, we use the electromagnetic calorimeters (ECAL) in the barrel and endcap region to look for photon candidates. A photon candidate is given by a "super cluster" (conglomerate of energy deposits, defined below) above some high transverse-energy threshold. They are obtained by using one of the standard $p+p$ clustering reconstruction schemes. For each photon candidate, we then examine the information provided by several shape variables of the clusters associated with the candidate. Together with the information from the hadronic calorimeters (HCAL), in the barrel and endcap region, as well as the Silicon Tracker, we determine if a given photon candidate is an isolated photon.

To select photon candidates we use the standard ECAL reconstruction procedures as outlined in Ref. [12].

To further characterize the photon candidates, we use cluster shape variables based on the ECAL energy distribution in the crystals assigned to a given cluster. Finally, for a given photon candidate, we inspect the local environment in a cone of $\Delta R$ with respect to the centroid of the super cluster for hadronic activity, including reconstructed tracks, in order to determine if a given veto threshold is reached. For the cone energy cuts, the underlying $\mathrm{Pb}+\mathrm{Pb}$ background energy is subtracted.

The cone energy and shape variables are separately combined into two joint variables, with coefficients determined by the Fisher linear discriminant method in the TMVA package in ROOT [13]. The combined cone energy and shape variables, in addition with the track isolation variables, then form a three-dimensional space, in which an optimal rectangular cut is again determined using the TMVA package to divide the candidate sample into signal (isolated photons) and background.

The obtained background rejection power as a function of signal efficiency, so called Receiver Operating Characteristics (ROC) curve, is shown in the top panel of Fig. 3.1 for quenched and unquenched $p+p$ and $0-10 \%$ central $\mathrm{Pb}+\mathrm{Pb}$ events. It should be emphasized that for both systems the coefficients and cuts of the corresponding unquenched training samples are used, i.e. for quenched $p+p(\mathrm{~Pb}+\mathrm{Pb})$ the values obtained from training with unquenched $p+p$ $(\mathrm{Pb}+\mathrm{Pb})$.

The transverse energy distribution of reconstructed isolated photons obtained at a working point of $60 \%$ signal efficiency is shown in Fig. 3.2 for $p+p$ (PYTHIA) and 0$10 \%$ central quenched $\mathrm{Pb}+\mathrm{Pb}$ (HYDJET). Application of the isolation and identification chain for photon candidates with $E_{\mathrm{T}}>70 \mathrm{GeV}$ leads to a signal-over-background ratio of about 12 (4.5) for the $p+p$ (quenched $\mathrm{Pb}+\mathrm{Pb}$ ) case, which corresponds to an improvement of about a factor of 100 (15) (see Fig. 3.3). ${ }^{1}$

On average, for $E_{\mathrm{T}}>70 \mathrm{GeV}$, the transverse energy resolution for isolated photons is about $4.5 \%$. Finally, it should
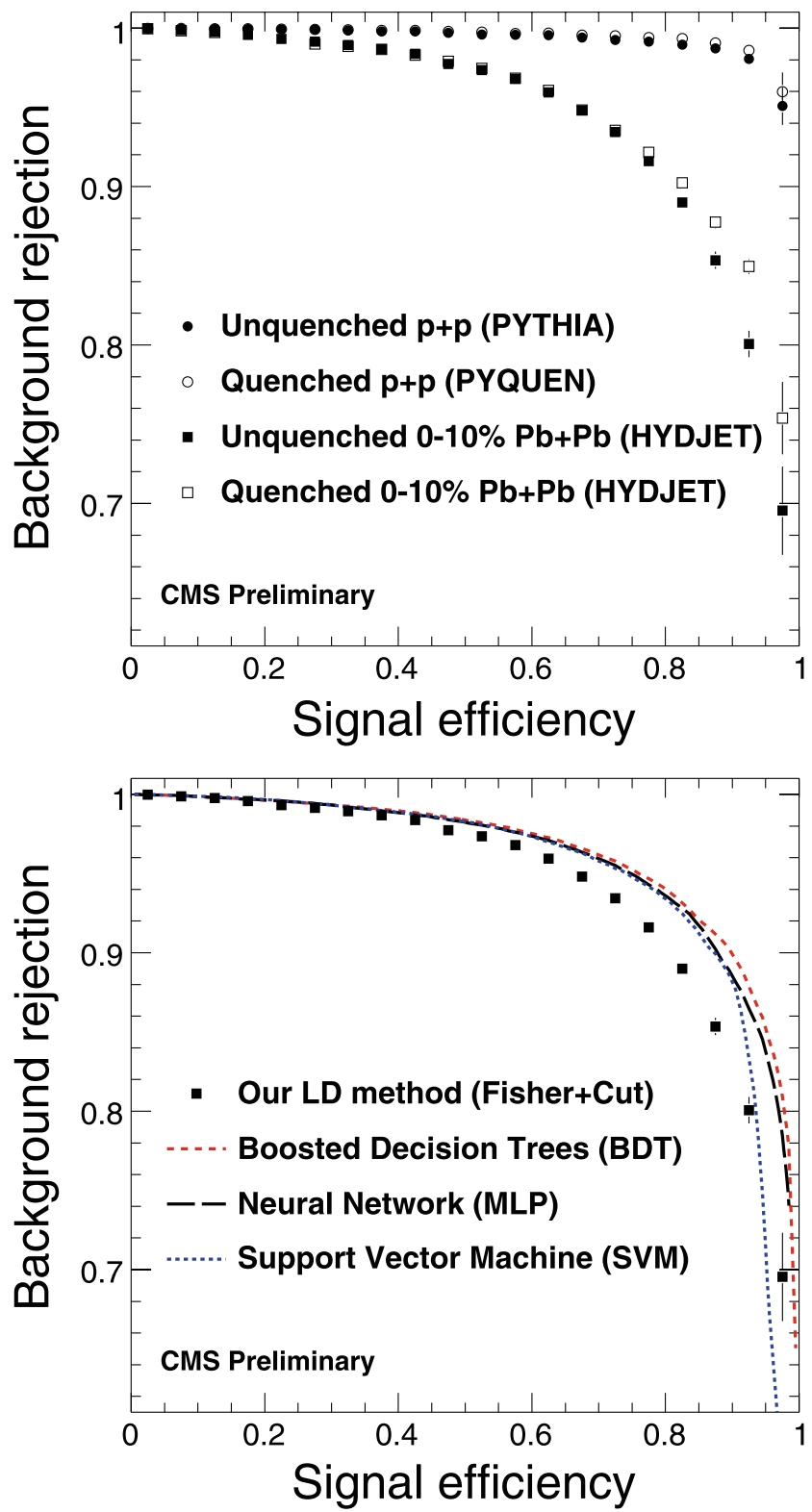

Fig. 3.1 Top panel: Background rejection vs signal efficiency for the identification of isolated photons compared for $p+p$ (PYTHIA), quenched $p+p$ (PYQUEN) and $0-10 \%$ central quenched and unquenched $\mathrm{Pb}+\mathrm{Pb}$ (HYDJET) events. In all cases the coefficients and cuts are obtained for the corresponding unquenched sample and applied to the quenched case. Bottom panel: Background rejection versus signal efficiency for $0-10 \%$ central $\mathrm{Pb}+\mathrm{Pb}$ (HYDJET) events obtained using several multivariate analysis techniques provided by TMVA compared with the Fisher + Cut method

\footnotetext{
${ }^{1} \mathrm{Also}$ in the unquenched $\mathrm{Pb}+\mathrm{Pb}$ case a factor of 15 improvement is reached, leading to $S / B \approx 1.5$.
} 

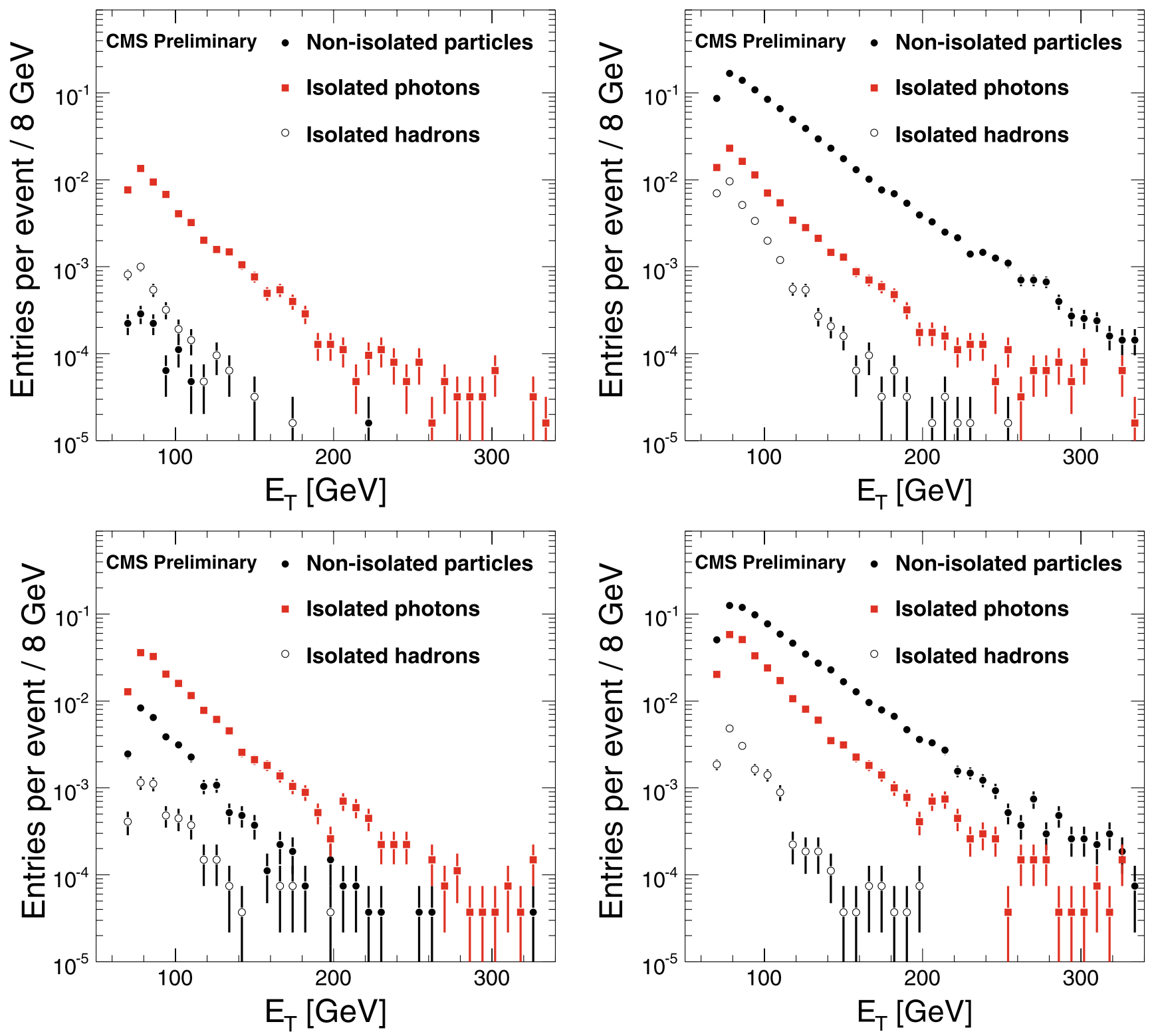

Fig. 3.2 Transverse energy distribution of reconstructed isolated photons (working point chosen at $60 \%$ signal efficiency) related to different types of trigger particles. Non-isolated particles, isolated photons and isolated hadrons are classified using the isolation algorithm on the MC truth. Shown are $p+p$ (PYTHIA, top panel) and $0-10 \%$ central quenched $\mathrm{Pb}+\mathrm{Pb}$ (HYDJET, bottom panel) events

be noted that the spatial resolution in $\eta$ and $\phi$ is better than 0.005 .

\section{Extracting fragmentation functions}

To extract fragmentation functions of parton initiated jets in $\gamma$-jet events, isolated photon candidates are selected and correlated with back to back reconstructed calorimeter jets in $\mathrm{Pb}+\mathrm{Pb}$ events.

The top panel of Fig. 4.2 shows the $\Delta \phi$ distribution between a photon and the away side parton selected at gen-

Fig. 3.3 Transverse energy distribution of photon candidate super clusters before application of reconstruction isolation cuts. The different types of generated particles, non-isolated particles, isolated photons and isolated hadrons, are classified using the isolation algorithm on the MC truth. Shown are $p+p$ (PYTHIA, top panel) and $0-10 \%$ central quenched $\mathrm{Pb}+\mathrm{Pb}$ (HYDJET, bottom panel) events

erator level (see Sect. 2.1). The vertical lines correspond to the back-to-back criterion defining valid signal events of $\Delta \phi>3$. For the selected photon jet pairs, reconstructed charged particles that lie within an 0.5 radius cone in $\eta-\phi$ around the reconstructed jet axis are selected. The fragmentation function is constructed using the transverse energy of the photon as a measure of the parton transverse energy and the reconstructed transverse momentum of the tracks in the cone. To extract the fragmentation function using the charged particle information obtained in the tracker, 
the away side jet associated with the photon needs to be contained in the tracker acceptance of $|\eta|<2.5$. For this analysis, jets with their axis within $|\eta|<2.0$ are selected to avoid edge effects at the limit of the tracker acceptance. The bottom panel of Fig. 4.2 shows the $\eta$ distribution of the away side partons associated with the signal photons entering this analysis. The vertical lines indicate the pseudorapidity range that can be selected for this analysis.

To reconstruct a photon jet correlation each photon candidate is associated with the highest $E_{\mathrm{T}}$ calorimeter jet fulfilling the back to back criterion of $\Delta \phi>3$. In order to en-
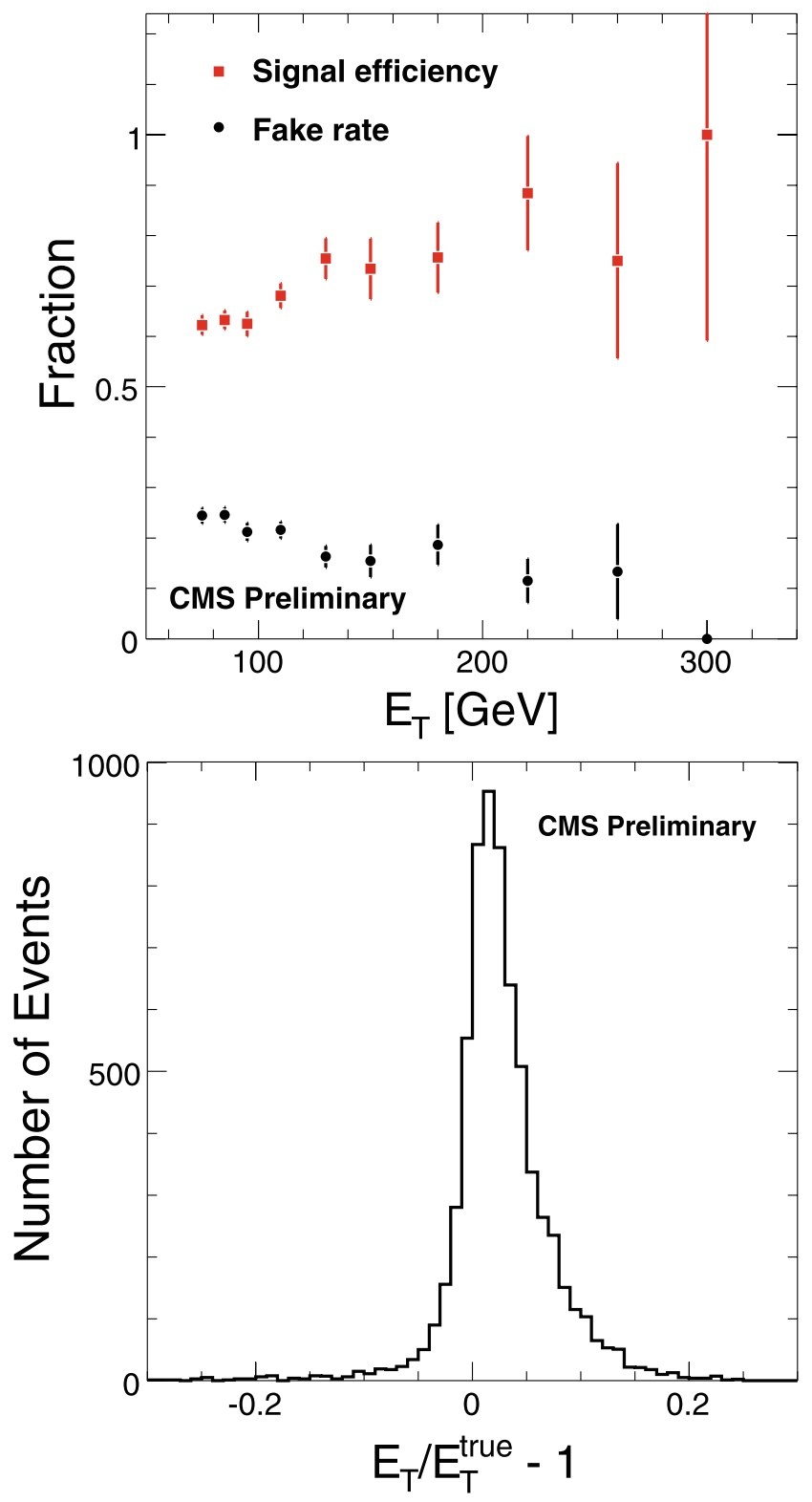

Fig. 4.1 Top panel: Efficiency (and fake rate) of reconstructed isolated photons as a function of reconstructed transverse energy in 0-10\% central quenched $\mathrm{Pb}+\mathrm{Pb}$ (HYDJET) events. Bottom panel: Energy response of reconstructed isolated photons with $E_{\mathrm{T}}>70 \mathrm{GeV}$. In both panels the working point is set to a signal efficiency of $60 \%$ sure that the reconstructed calorimeter jet corresponds to the away side parton a minimum jet $E_{\mathrm{T}}$ cutoff is applied.

For reconstructable $\gamma$-jet pairs the efficiency to reconstruct the away side jet is estimated by requiring a reconstructed calorimeter jet and matching the associated away side parton by requiring a difference in $\eta$ of less than 0.2 . The top panel of Fig. 4.3 shows the efficiency to reconstruct findable $\gamma$-jet pairs in case of quenched jets as a function of the $\gamma$ transverse energy. The bottom panel shows the efficiency as a function of the calorimeter jet energy.

For this analysis all photon candidate ECAL clusters are used which pass the isolation cuts. For each event the highest
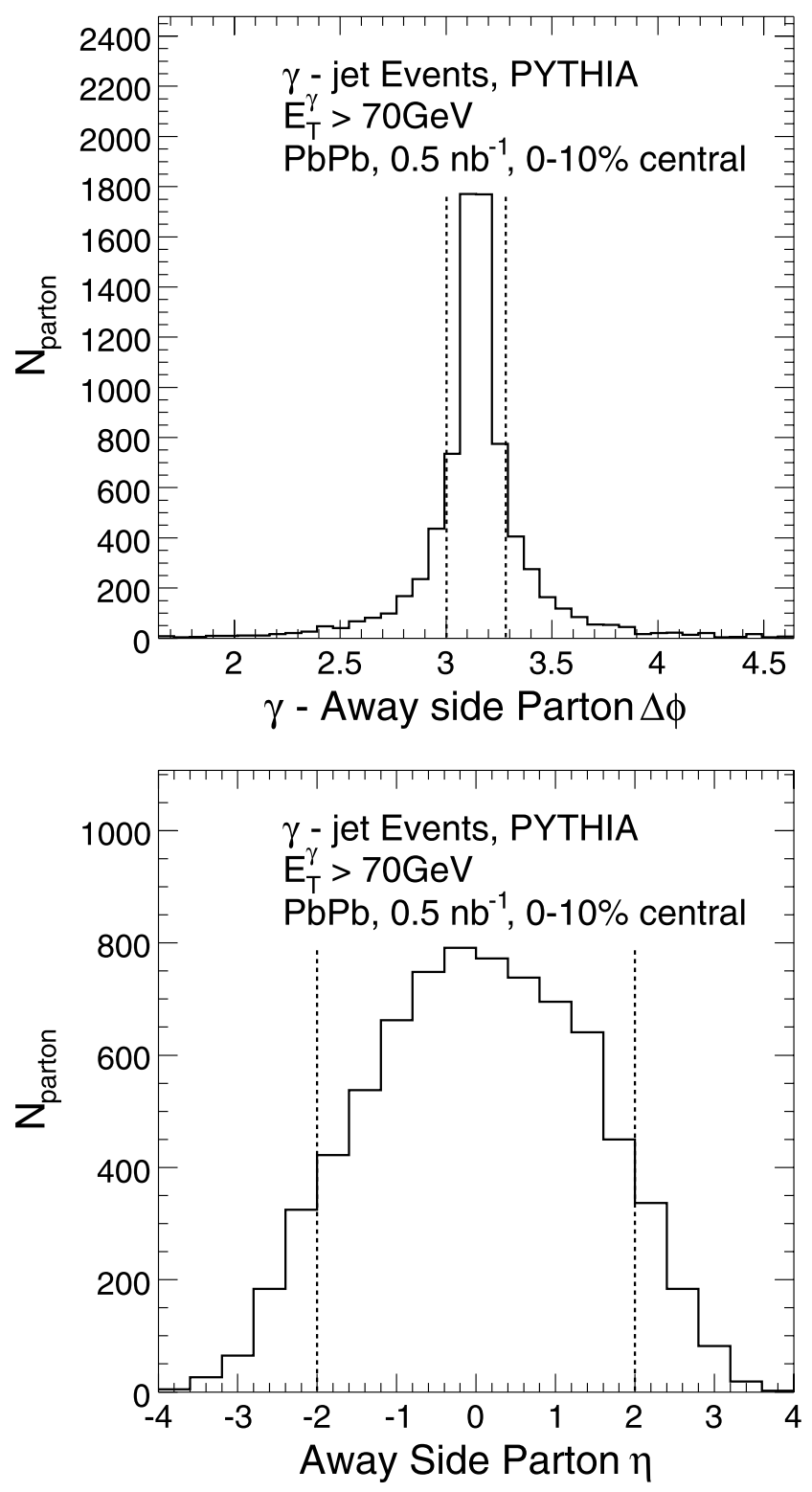

Fig. 4.2 Top panel: Opening angle distribution between isolated photons and the associated away side parton for photons with a transverse momentum $p_{\mathrm{T}}>70 \mathrm{GeV} / c$. Bottom panel: $\eta$ distribution of the away side parton associated with isolated photons selected for this analysis 

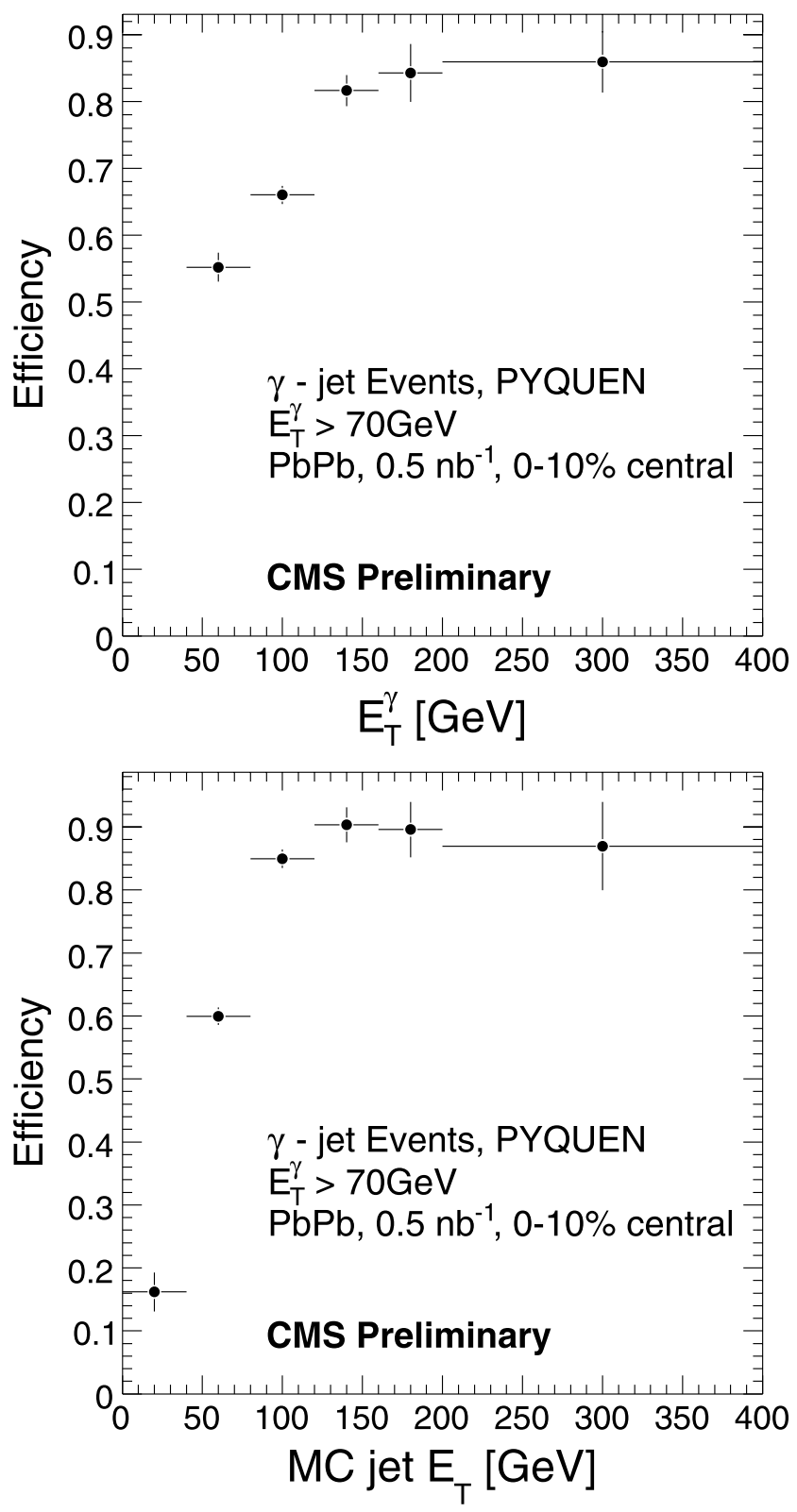

Fig. 4.3 Top panel: Reconstruction efficiency for findable quenched $\gamma$-jet pairs as a function of the $\gamma$ transverse energy. Bottom panel: Reconstruction efficiency of findable $\gamma$-jet pairs as a function of the away side jet energy

$E_{\mathrm{T}}$ ECAL cluster is selected as a photon candidate. For the purpose of this analysis we require a minimum $E_{\mathrm{T}}$ of the ECAL cluster of 70 (100) GeV.

\subsection{Underlying event subtraction}

To extract the fragmentation functions of parton-induced jets in the $\gamma$-jet channel in central $\mathrm{Pb}+\mathrm{Pb}$ events special care needs to be taken to estimate the underlying event contribution from the copious soft background of the $\mathrm{Pb}+\mathrm{Pb}$ events. The underlying event contribution is estimated by using the momentum distributions of tracks found in an area outside of the jet cone, which is assumed to only contain background particles. For this purpose, for each reconstructed isolated ECAL cluster-jet pair the charged tracks within a 0.5 radius cone perpendicular in $\phi$ angle to the reconstructed jet axis are selected. This selection of particles is free of signal particles and, using the ECAL cluster transverse energy, the selected particles can be used to obtain the contribution to the fragmentation function by the underlying $\mathrm{Pb}+\mathrm{Pb}$ event.

\subsection{Systematic uncertainties}

We determined the contribution from three main sources to the difference between reconstructed and true fragmentation functions. These sources are:

- Contributions of QCD jet fragmentation products misidentified as isolated photons.

- Association of a wrong/fake jet on the away side of the isolated photon.

- Biases due to jet reconstruction at low jet $E_{\mathrm{T}}$.

The latter effect gives the largest contribution to the estimated systematic uncertainty of this analysis, in particular for the quenched scenario.

The low jet reconstruction efficiency for low $E_{\mathrm{T}}$ jets potentially leads to a bias in the reconstructed fragmentation function. At low $E_{\mathrm{T}}$, jets fragmenting into single high $p_{\mathrm{T}}$ particles are more likely to be reconstructed. This results in a bias in the measurement to harder fragmentation functions. The systematic error of the measurement associated with the bias of the reconstructed fragmentation functions due to the jet finder efficiency is the dominant source of uncertainty for this measurement, in particular for the lower photon energy selection.

QCD jet fragmentation products that pass the ECAL cluster isolation cuts are misidentified as isolated photons.

The isolation process requires little hadronic energy contained in a cone around these particles, so this biases the fraction of the original parton momentum these particles carry from the average of about 0.3 for leading particles in a jet closer to unity. Still these high $z=p_{\mathrm{T}} / E_{\mathrm{T}}^{\text {parton }}$ particles underestimate the energy of the associates away side parton. This results in observing a harder fragmentation function compared to the true fragmentation function when using the $E_{\mathrm{T}}$ of these particles to trace the away side parton $E_{\mathrm{T}}$.

Associating a wrong/fake jet on the away side of the isolated photon will results in observing a softer fragmentation function since it samples low $E_{\mathrm{T}}$ jets from fluctuations in the soft background. This effect can also be studied based on MC truth information by comparing reconstructed fragmentation functions for all events to ones constructed only for good matches of the reconstructed jets to the true away side parton. 


\section{Results and discussion}

The fully reconstructed fragmentation functions included underlying event subtraction are shown in Fig. 5.1 using a $70 \mathrm{GeV}$ ECAL cluster energy cutoff and in Fig. 5.2 using a $100 \mathrm{GeV}$ cutoff. The top panel in each figure shows the results for the unquenched (PYTHIA) scenario, while the bottom panel shows the result for a model including jet-quenching (PYQUEN). The estimated systematic error
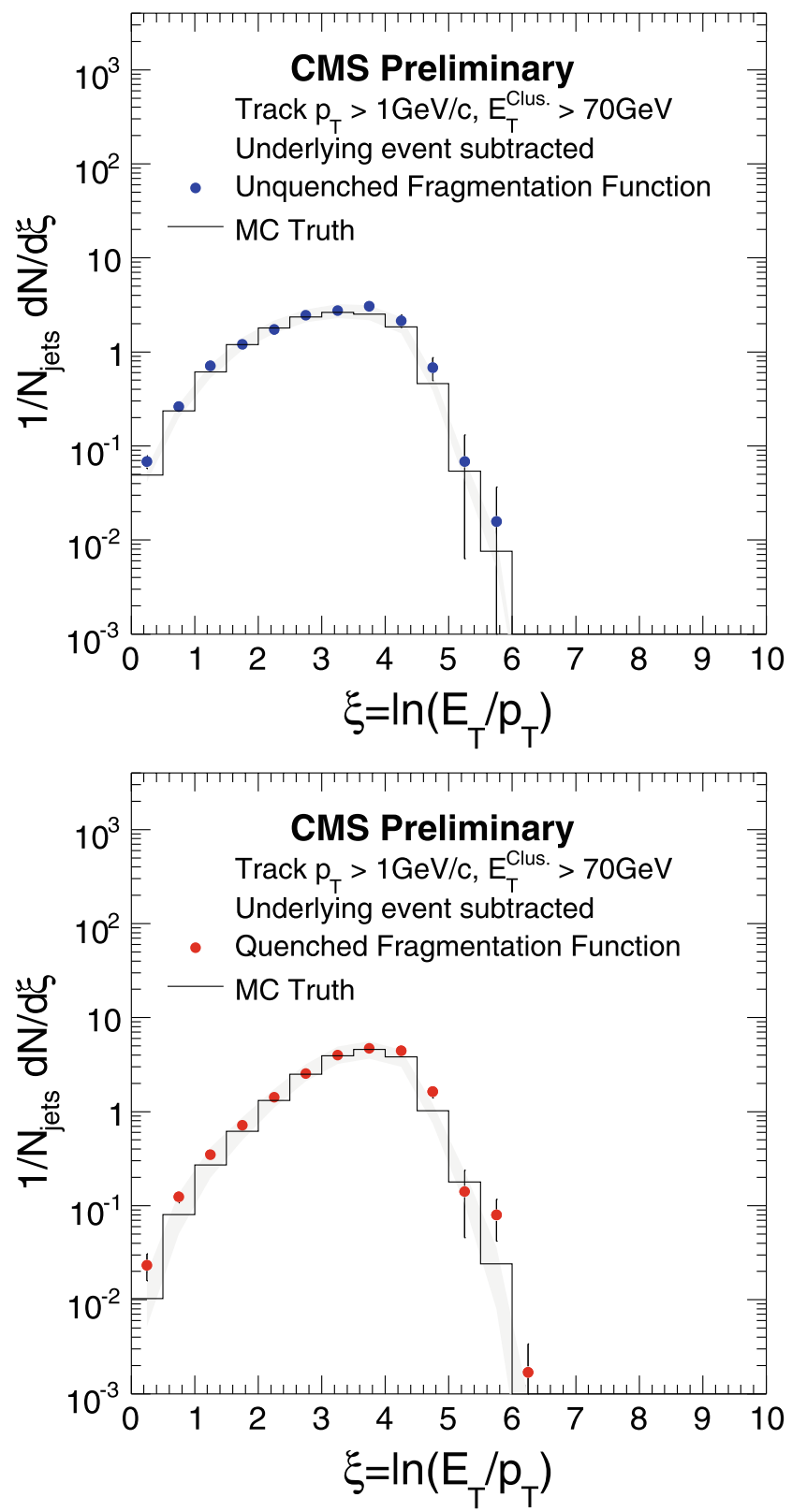

Fig. 5.1 Underlying event subtracted fragmentation functions extracted from central $\mathrm{Pb}+\mathrm{Pb}$ collisions (symbols) and $\mathrm{MC}$ truth signal fragmentation function. ECAL Cluster $E_{\mathrm{T}}>70 \mathrm{GeV}$. Top panel: unquenched jets (PYTHIA), unquenched underlying event (HYDJET) subtracted. Bottom panel: quenched jets (PYQUEN), quenched underlying event (HYDJET) subtracted of the measurement is represented as the shaded band in the figures. The reconstructed fragmentation functions are overlaid with the MC truth fragmentation functions determined at generator level using the true parton $E_{\mathrm{T}}$ and direction for selection of particles. The true fragmentation functions extracted from the generator level information are very well reproduced by the reconstructed fragmentation functions based on the photon, jet and charged particle reconstruction, for both scenarios and for both photon energy ranges.
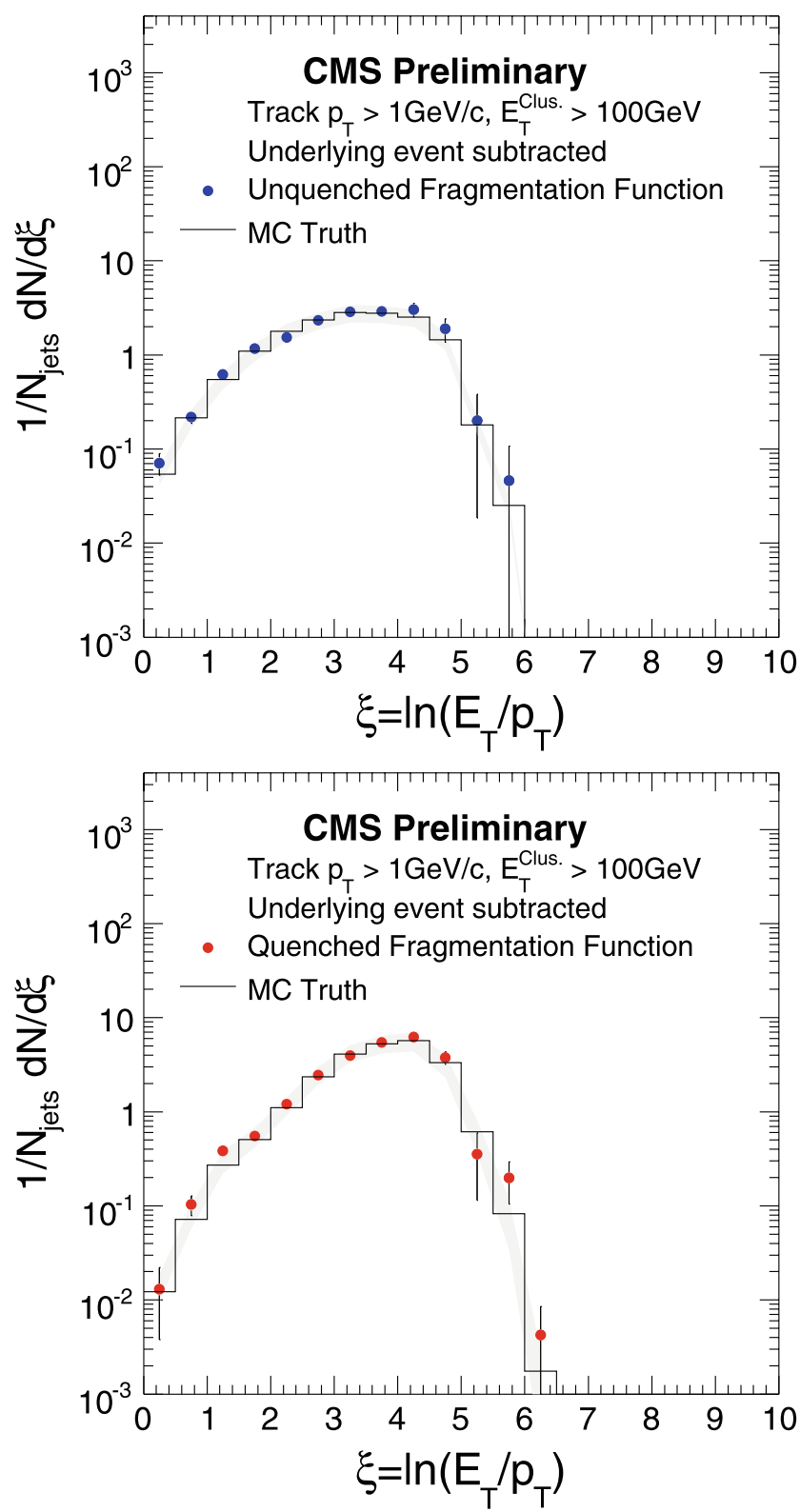

Fig. 5.2 Underlying event subtracted fragmentation functions extracted from central $\mathrm{Pb}+\mathrm{Pb}$ collisions (symbols) and $\mathrm{MC}$ truth signal fragmentation function. ECAL Cluster $E_{\mathrm{T}}>100 \mathrm{GeV}$. Top panel: unquenched jets (PYTHIA), unquenched underlying event (HYDJET) subtracted. Bottom panel: quenched jets (PYQUEN), quenched underlying event (HYDJET) subtracted 
To further illustrate the quality of reconstruction, the ratio of the reconstructed fragmentation functions over the MC truth fragmentation functions are shown in Fig. $5.3(70 \mathrm{GeV}$ cutoff) and Fig. 5.4 (100 GeV cutoff) for unquenched and quenched scenarios.

For both photon energy ranges and both scenarios, the reconstructed and truth fragmentation functions agree within the statistical and estimated systematic uncertainties. This is in particular true for the intermediate $\xi$ range. The com-
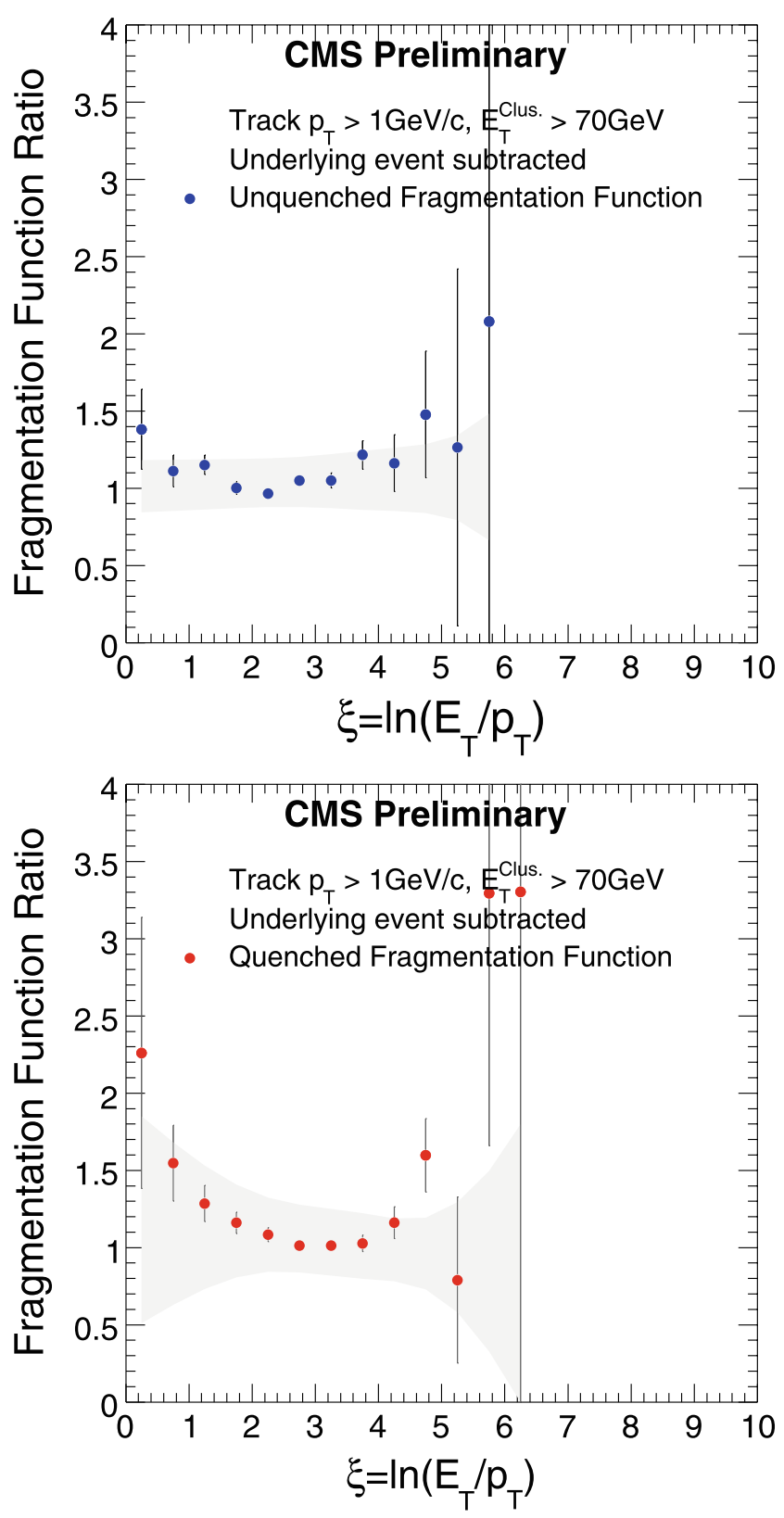

Fig. 5.3 Underlying event subtracted fragmentation functions extracted from central $\mathrm{Pb}+\mathrm{Pb}$ collisions divided by the MC truth signal fragmentation function. ECAL Cluster $E_{\mathrm{T}}>70 \mathrm{GeV}$. Top panel: unquenched jets (PYTHIA), unquenched underlying event (HYDJET) subtracted. Bottom panel: quenched jets (PYQUEN), quenched underlying event (HYDJET) subtracted parison of the $E_{\mathrm{T}}>70 \mathrm{GeV}$ and $E_{\mathrm{T}}>100 \mathrm{GeV}$ selection illustrate the trade-off between statistical and systematic uncertainties for the two photon energy ranges. As mentioned previously, the particular implementation of the energy loss mechanism in PYQUEN amplifies the effect of the jet finding efficiency, as the energy lost by the parton is radiated out of the typical jet cone area. Further optimization of the jet finding algorithm or simulation based corrections for the
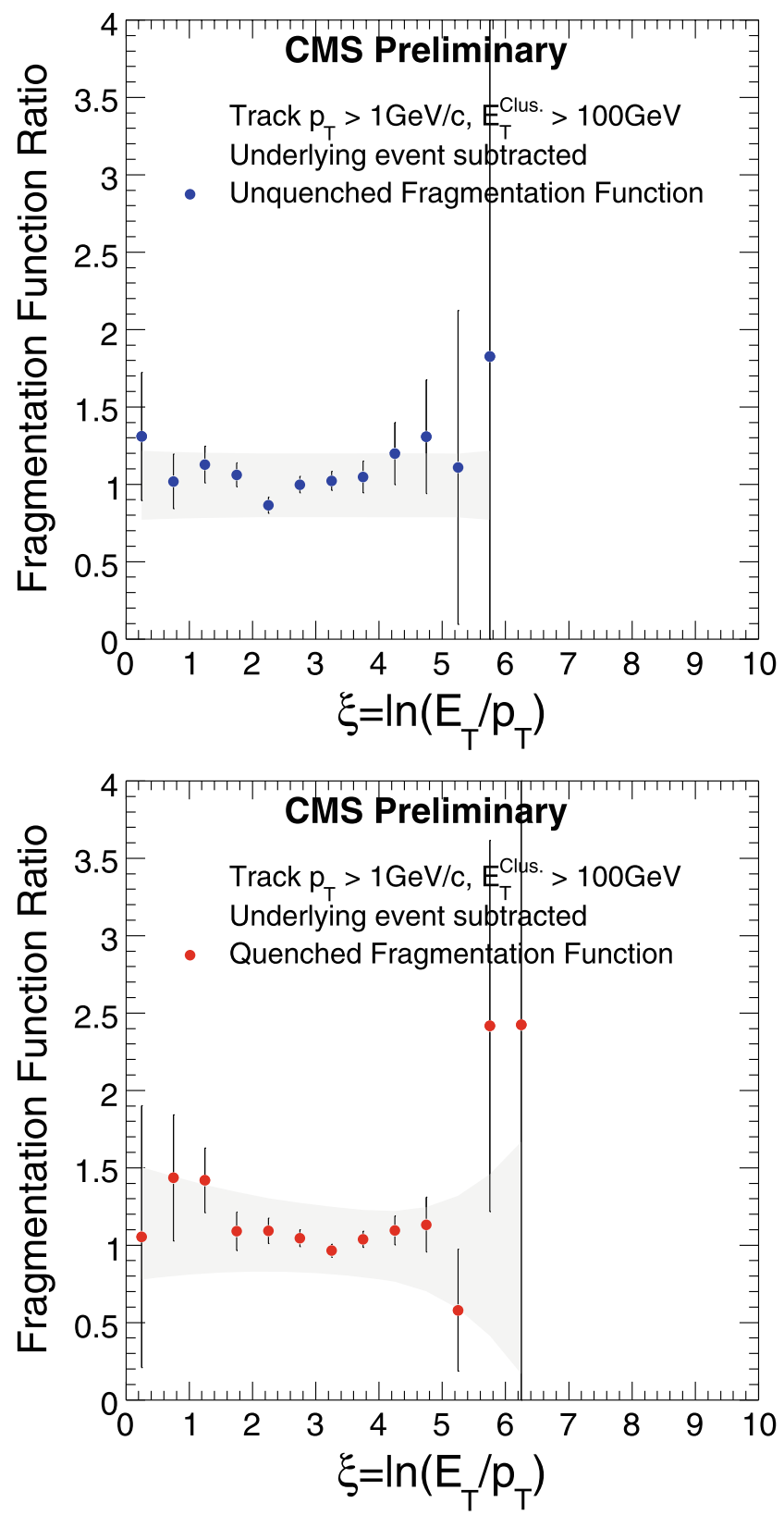

Fig. 5.4 Underlying event subtracted fragmentation functions extracted from central $\mathrm{Pb}+\mathrm{Pb}$ collisions divided by the $\mathrm{MC}$ truth signal fragmentation function. ECAL Cluster $E_{\mathrm{T}}>100 \mathrm{GeV}$. Top panel: unquenched jets (PYTHIA), unquenched underlying event (HYDJET) subtracted. Bottom panel: quenched jets (PYQUEN), quenched underlying event (HYDJET) subtracted 
jet finder bias should allow a reduction of the current systematic uncertainties.

The overall capability to measure the medium-induced modification of jet fragmentation functions in the $\gamma$ jet channel can be illustrated by comparing the fully reconstructed quenched fragmentation function to the unquenched MC truth distribution. The change in the fragmentation function between the unquenched and quenched case provides
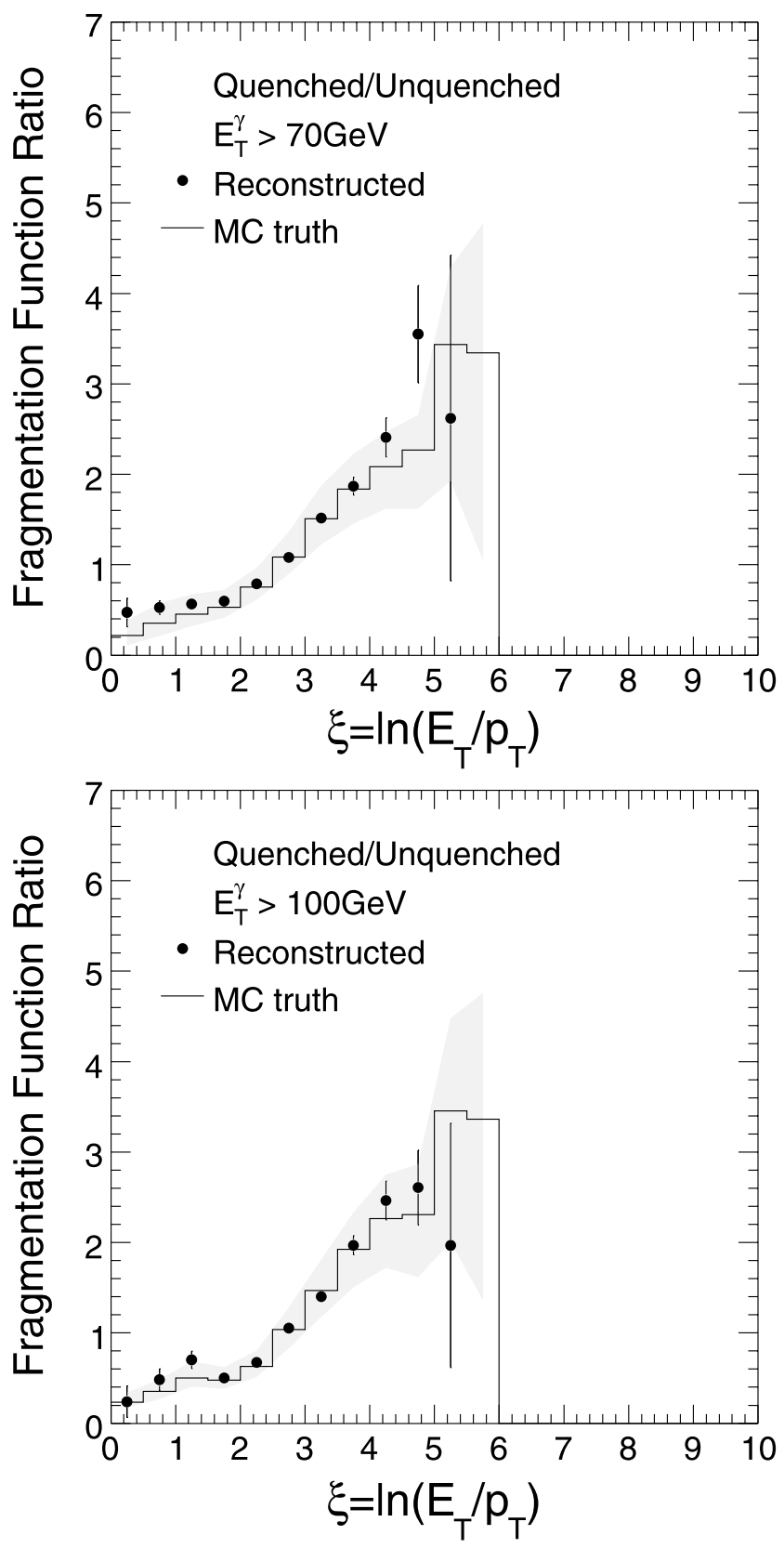

Fig. 5.5 Top panel: Ratio of the reconstructed quenched fragmentation function for events using a minimum $E_{\mathrm{T}}$ for the photon candidate ECAL cluster of $70 \mathrm{GeV}$ (symbols). Ratio of the corresponding MC truth fragmentation functions (line). Bottom panel: Ratio of the reconstructed quenched fragmentation function for events using a minimum $E_{\mathrm{T}}$ for the photon candidate ECAL cluster of $100 \mathrm{GeV}$ (symbols). Ratio of the corresponding MC truth fragmentation functions (line) the scale against which the observed uncertainties can be judged.

Figure 5.5 shows the ratio for the reconstructed quenched fragmentation function (symbols) and the corresponding MC truth ratio (line). The top panel shows the ratio for a $70 \mathrm{GeV}$ ECAL cluster cut and bottom panel for $100 \mathrm{GeV}$. One sees that the ratio of reconstructed fragmentation functions to the unquenched MC truth tracks the same ratio in the MC truth over the full $\xi$-range for both $E_{\mathrm{T}}$-ranges within the estimated uncertainties. For essentially the full $\xi$ range, the measurement uncertainties are much smaller than the change in the fragmentation function caused by the jetquenching effect.

\section{Conclusions}

In summary, we have shown that $\gamma$-jet events can be used to study the medium-modifications of the fragmentation functions of high $E_{\mathrm{T}}$ partons quantitatively. For a data set corresponding to a nominal one-month $\mathrm{LHC} \mathrm{Pb}+\mathrm{Pb}$ run, the expected statistical and systematic uncertainties are small compared to the expected change in the fragmentation functions relative to parton fragmentation in vacuum. This measurement will allow a quantitative test of proposed mechanisms for parton energy loss in the medium, testing fundamental properties of the high density QCD medium produced in high energy nuclear collisions.

\section{References}

1. I. Arsene et al. (BRAHMS Collaboration), Nucl. Phys. A 757, 1 (2005). arXiv:nucl-ex/0410020

2. B.B. Back et al. (PHOBOS Collaboration), Nucl. Phys. A 757, 28 (2005). arXiv:nucl-ex/0410022

3. K. Adcox et al. (PHENIX Collaboration), Nucl. Phys. A 757, 184 (2005). arXiv:nucl-ex/0410003

4. J. Adams et al. (STAR Collaboration), Nucl. Phys. A 757, 102 (2005). arXiv:nucl-ex/0501009

5. X.N. Wang, Z. Huang, Phys. Rev. C 55, 3047 (1997). arXiv: hep-ph/9701227

6. I.P. Lokhtin, A.M. Snigirev, Eur. Phys. J C16, 527 (2000)

7. Code is adapted from http://cmssw.cvs.cern.ch/cgi-bin/cmssw. cgi/sCMSSW/GeneratorInterface/GenFilters/src/PythiaFilter GammaJet.cc?revision=1.3\&view=markup\&pathrev =CMSSW_1_5_4

8. G. Baur et al., Heavy ion physics program in CMS, CMSNOTE/2000-060

9. CMS Collaboration, High density QCD with heavy ions, CERN/LHCC 2007-009

10. C. Roland et al., CMS-NOTE/2006-031, 2006

11. CMS Collaboration, The tracker project technical design report, CERN/LHCC 98-006. CMS TDR 5, Addendum CERN/LHCC 2000-016

12. CMS Collaboration, Physics technical design report, CERN/LHCC 2006-001

13. Toolkit for Multivariate Data Analysis with ROOT, v3.8.11. http://tmva.sourceforge.net/ 